\title{
Zur Nützlichkeit des Morphem-Begriffes in der Deutschlehrerausbildung (DaF)
}

\section{Roland Wagner}

The article argues against the use of the concept "morpheme" in introductory classes to linguistics aimed at students of German as a foreign language within teaching degree programs. The use of more neutral terminology as "affix" or "stem" is recommended. The main reasons for the suggestion are (a) notorious problems in applying morpheme-based systems to German morphology (e.g. "Ablaut"), (b) developments inside linguistics within the last 40 years, which led to a loss of significance of the morpheme-based approach to language, (c) irrelevance of the concept for using reference grammars and handbooks in the field of German as a foreign language.

Introduction to linguistics - linguistic terminology - morpheme - morph - German as a foreign language - German morphology

Im vorliegenden Artikel werden Gründe gegen die Verwendung des Begriffs „Morphem“ in Einführungskursen in die Linguistik angeführt, die im Rahmen von Lehramtsstudiengängen im Fach „Deutsch“im nichtdeutschsprachigen Ausland durchgeführtwerden. Diehauptsächlichen Gründe sind: (a) notorische Schwierigkeiten bei der Anwendung von morphembasierten Analysen auf deutsches Sprachmaterial (z. B. beim „Ablaut“), (b) Entwicklungen innerhalb der Linguistik der letzten 40 Jahre, die die strukturalistischen, morphembasierten Ansätze aus ihrer früheren Führungsposition verdrängt haben, (c) fehlende Relevanz des Begriffes für die Benutzung von Standardwerken und Handbüchern des DaF-Unterrichts. Statt „Morphem“ wird die Verwendung theoretisch neutraler Begriffe wie „Stamm“ oder „Affix“ empfohlen.

Einführung in die Linguistik - linguistische Terminologie - Morphem - Morph - Deutsch als Fremdsprache - deutsche Morphologie

Morphem, Morph und Allomorph sind also in einem Handbuch der Morphologie weniger deshalb zu behandeln, weil es so etwas wie Morpheme, Morphe und Allomorphe in den Sprachen der Welt objektiv gibt, sondern weil man in der Sprachwissenschaft seit vielen Jahrzehnten mit diesen Konzepten gearbeitet hat.

(Luschützky 2000: 451)

An vielen Hochschulen in Mitteleuropa ${ }^{1}$ gehört der Begriff "Morphem“ heute ins Standardrepertoire der Fachbegriffe, mit denen Studienanfänger in sprachwissenschaftlichen Studiengängen vertraut gemacht werden. Dies gilt u. a. auch für Studiengänge, in denen zukünftige Fremdsprachenlehrer für die

1 Konkrete Erfahrungen mit Einführungskursen in die Sprachwissenschaft hat der Autor als Lektor an der Pädagogischen Fakultät der Masaryk-Universität in Brünn gemacht. Aufgrund dieser Erfahrungen ist auch der vorliegende Artikel entstanden. 
Grundschulen ${ }^{1}$ ausgebildet werden, so z. B. an der Masaryk-Universität in Brünn/ Tschechische Republik (vgl. etwa die hier verwendeten Skripten Káňa 2006 und Geislerová 2007).

Nun ist die Vermittlung von Fachbegriffen kein Wert an sich. Begriffe sind Werkzeuge, mit denen sich der menschliche Geist die Welt erschließt. Bringt ein Begriff für den jeweiligen konkreten Zweck, zu dem er eingesetzt wird, keinen Erkenntniszuwachs, handelt es sich um ein stumpfes Werkzeug, das geschärft oder aber ausgetauscht werden muss. Auf den folgenden Seiten möchte ich ein paar Überlegungen dazu anstellen, ob der Morphembegriff für die Zwecke von Deutschlernern in Tschechien, die das Lehramt an dortigen Grundschulen anstreben, ein brauchbares Werkzeug darstellt. Dabei ist zu fragen, (a) ob der Morphembegriff den Studierenden zu einem besseren Verständnis der fremdsprachlichen Strukturen verhilft oder ihnen gar die Aneignung solcher Strukturen erleichtert, (b) ob die Kenntnis dieses Begriffes Voraussetzung für die selbständige Lektüre von Fachliteratur ist und somit eine Voraussetzung für das lebenslange Lernen, heute (zusammen mit der Unterstützung der Lernerautonomie) ein wichtiges Bildungsziel an öffentlichen Hochschulen (vgl. z. B. Janíková 2012).

Die eben angedeutete Fragestellung muss klar von einer linguistischen Fragestellung im engeren Sinne abgegrenzt werden, die die Brauchbarkeit des Morphembegriffs für die Beschreibung und Erklärung sprachlicher Strukturen überhaupt zum Gegenstand hätte. Fragen der linguistischen Brauchbarkeit des Morphembegriffs werden daher nur am Rande (in Abschnitt 2) gestreift, um einen Hintergrund für die praxisbezogene Diskussion in Abschnitt 3 und 4 zu haben.

\section{Genealogie}

Die Einführung des Begriffs „Morphem“ zur Bezeichnung von unteilbaren Spracheinheiten mit Bedeutungen wird gewöhnlich Baudouin de Courtenay (1880) zugeschrieben, auch wenn der Autor den Begriff noch recht uneinheitlich verwendet hat (vgl. Mugdan 1986: 30-31; Anderson 1992: 11). Sowohl Mugdan als auch Anderson weisen auf den Zusammenhang von Baudouins terminologischer Innovation mit dem semiotischen Sprachverständnis hin, das sich später am klarsten in de Saussures Cours de linguistique générale (1916; 2. Aufl. 1931) niederschlagen sollte. Da die Vorstellung, Sprache sei ein Zeichensystem, vielen Lesern von heute geradezu als banale Selbstverständlichkeit erscheinen mag, muss an dieser Stelle kurz die ursprüngliche Pointe des Zeichengedankens rekonstruiert werden.

Die sprachwissenschaftliche Epoche, die dem Cours unmittelbar vorangegangen war, betrachtete sprachliche Einheiten in erster Linie aus Sicht ihrer Entstehung.

1 Mit dem Begriff „Grundschule“ beziehe ich mich auf das mir vertraute Schulsystem in Tschechien, wo die Grundschule die Primarstufe I und die Sekundarstufe I (1. bis 9. Jahrgangsstufe) umfasst. 
Eine Form in Sprache L ist aus dieser Perspektive immer dann ein linguistisches Objekt, wenn sich für diese Form auf einer früheren Sprachstufe von L (oder in einer verwandten Sprache L') eine entsprechende Form identifizieren lässt, die durch die zu analysierende Form fortgesetzt wird. So ist (wie noch heute in manchen Grammatiken des Deutschen nachzulesen, z. B. Erben 1972: 65; Helbig/ Buscha 1998: 49) ${ }^{1}$ das Verb sprengen das Kausativum zu springen, weil ersteres auf eine Verbindung der Wurzel sprang- mit dem Kausativsuffix -ja- zurückgeht, die - gemäß der lautgesetzlichen Entwicklung - im heutigen Deutschen die genannte Form liefert.

Eine radikal andere Sichtweise auf Sprache findet sich in de Saussures Cours unter der Überschrift, Synchronie“. Aus synchronerSicht ist Sprache ein System ausZeichen, die im Bewusstsein der Sprecher dieser Sprache durch dauerhafte Assoziation eines akustischen Bildes (einer Lautform) mit einer bestimmten Vorstellung konstituiert werden. Nur dann, wenn einem Lautkomplex im Bewusstsein des Sprechers eine bestimmte Vorstellung entspricht, handelt es sich bei diesem Lautkomplex um ein Zeichen und damit um ein sprachliches Objekt. ${ }^{2}$ Ausschlaggebend für die Analyse von sprachlichen Strukturen ist daher nicht der Vergleich mit früheren Sprachstufen derselben Sprache (bzw. der Vergleich mit anderen, verwandten Sprachen), sondern allein das Urteil des kompetenten Sprechers:

La synchronie ne connaît qu'une perspective, celle des sujets parlants, et toute sa méthode consiste à recueillir leur témoignage; pour savoir dans quelle mesure une chose est une réalité, il faudra et il suffira de rechercher dans quelle mesure elle existe pour la conscience des sujets. (de Saussure 1931: Première Partie, Kap. III, $\$$ 5, S. 128)

Aus dem Gesagten folgt für die Analyse der Form sprengen, dass es sich keineswegs um ein „Kausativum“ zu springen handelt, auch wenn die historisch-vergleichende Sprachwissenschaft die Verbindungslinien zu urspr. Kausativbildungen aufzeigen kann: Weder enthält die Form einen Lautkomplex, den ein Sprecher des heutigen Deutschen mit der Bedeutungskomponente ,bewirken' assoziieren würde (vgl. etwa das Fehlen eines hypothetischen Kausativum klengen ,bewirken, dass etwas klingt ${ }^{t}$

1 Bei Helbig/Buscha (1998: 49) heißt es dazu wörtlich: „Das regelmäßige Verb [innerhalb der Verbvariante] ist jeweils kausativ, transitiv und hat einen Aktanten mehr, das entsprechende unregelmäßige Verb ist intransitiv". Die Beispielsätze lauten: Man sprengt den Felsen $\times$ Der Felsen sprang. Erben (1972: 65) macht immerhin explizit darauf aufmerksam, dass es sich im heutigen Deutschen nicht mehr um eine produktive Kategorie handelt.

2 Vgl. z. B. de Saussure (1931: Introduction, III, \$2, S. 32): La langue „[...] c’est un système de signes où il n'y a d'essentiel que l'union du sens et de l'image acoustique [....".

3 Die Synchronie kennt nur eine einzige Perspektive, und zwar die des sprechenden Subjekts. Die synchrone Methode erschöpft sich demgemäß darin, die Zeugnisse dieses Subjektes zu sammeln. Um zu wissen, in wie weit ein [sprachliches] Objekt eine Realität ist, ist es notwendig und hinreichend festzustellen, in wie weit dieses Objekt für das Bewusstsein des sprechenden Subjekts existiert. (Übersetzung R. W.) 
zum Verb klingen), noch ergibt sich im Bewusstsein eines solchen Sprechers eine Assoziation zwischen den Formen sprengen und springen, die eine paradigmatische Relation begründen könnte. Aus synchroner Sicht sind springen und sprengen einfach zwei verschiedene Verben mit verschiedener lexikalischer Bedeutung.

De Saussure selbst (1931: 109) demonstriert die Abgrenzung von synchroner, auf das Zeichen ausgerichteter Linguistik und diachroner Sprachwissenschaft an einem anderen Beispiel aus dem Deutschen: Die Form Drittel geht, wie bekannt, auf eine Verbindung des Zahlwortes für ,drei' mit der Wortform Teil hervor: Drittel $<{ }^{*}$ dritteil. Die Beziehung von -tel und Teil ist im heutigen Deutschen allerdings kein semiotisches Faktum mehr, da kompetente Sprecher des Deutschen keine Assoziation zwischen beiden Lautketten herstellen. Die Folge ist der Verlust der inneren Form von Drittel: Aus synchroner Sicht handelt es sich um ein unteilbares Minimalzeichen; „c'est un mot simple“, wie de Saussure schreibt.

Die Anwendung des Zeichenbegriffs bei der Bestimmung sprachlicher Einheiten ist das Moment, das den Begriff „Morphem“ “1 von älteren Vorläufern wie „Wurzel“, „Stamm“, „Affix“ oder „Formans“ unterscheidet. Morpheme sind die im Sprachmaterial vorfindbaren Lautketten nur dann, wenn sich die materielle Seite mit einer Bedeutung verbindet. Diese Auffassung spiegelt sich klar in den heute gängigen Morphemdefinitionen wieder. So heißt es etwa im Lexikon der Sprachwissenschaft von Bußmann, Morpheme seien die „[...] kleinste[n] bedeutungstragende[n] Elemente der Sprache, die als phonologisch-semantische Basiselemente nicht mehr in kleinere Elemente zerlegt werden können" (Bußmann 1990: 502). Ähnlich definiert Čermák: „Morfém je minimální významový nebo/a gramaticky distinktivní prvek, chápaný - v př́ípadě existence alomorfů - jako tř́́da alomorfü; ${ }^{2}$ je to základní prvek morfologie, který však má řadu forem a funkcí (Čermák 2011: 15).

\section{Linguistische Problematik}

Die Forschung der 40er und 50er Jahre, die hier nicht im Einzelnen referiert werden kann (vgl. aber z. B. Nida 1948; Hockett 1961), hat dann Schritt für Schritt die Schwierigkeiten offenbar werden lassen, auf die das Konzept vom Morphem als der kleinsten bedeutungstragenden Einheit bei Anwendung auf konkretes

1 Um Missverständnissen vorzubeugen sei darauf hingewiesen, dass de Saussure selbst den Begriff "Morphem" (meines Wissens) nicht verwendet hat.

2 Schon Lamb (1966:33) hat gezeigt, dass die Vorstellung vom Morphem als Menge von Allomorphen (aufgrund mengenlehretheoretischer Überlegungen) „unhaltbar" ist: Mengen, die leere Elemente (Null-Allomorphe) enthalten, führen den Mengenbegriff ad absurdum. Lamb selbst zieht daraus die Konsequenz, dass ein adäquates Sprachmodell nicht auf der Relation Element - Menge, sondern auf dem Begriff der Repräsentation aufgebaut werden muss. Sprache gliedert sich demnach in verschiedene Ebenen („Strata“), deren Elemente zueinander im Verhältnis der Repräsentation stehen. 
Sprachmaterial stößt. Für ein besseres Verständnis der Problematik scheint es mir an dieser Stelle nützlich zu sein, von der idealtypischen Ausprägung des Modells ${ }^{1}$ auszugehen und die Implikationen eines maximalen Morphembegriffes aufzuzeigen, die zwar je nach empirischer Zielsetzung abgeschwächt werden können, dann aber gleichzeitig mit einer Schwächung des Morphembegriffes verbunden sind. Ich folge dabei der Diskussion in Matthews (1972, Kap. 4), die meiner Ansicht nach für eine Klärung der verworrenen Verhältnisse besonders geeignet ist.

Matthews macht drei verschiedene Behauptungen explizit, die implizit in einem auf dem Morphembegriff fußenden linguistischen Modell enthalten sind:

(i) Die Zuordnung von Laut und Bedeutung erfolgt bis hinab zur kleinsten Einheit in diskreten Schritten. Jedem Segment auf der Ausdrucksseite entspricht immer genau eine Bedeutungseinheit auf der Inhaltsseite, und umgekehrt: Jeder Bedeutungseinheit ist immer ein bestimmtes Segment auf der Ausdrucksseite zugeordnet. Dabei ist es gleichgültig, wie groß die "Strukturportionen“ sind, die man dem Material entnimmt, vorausgesetzt, man unterschreitet nicht die Morphemgrenze. Mit anderen Worten: Bedeutung und Form verhalten sich isomorph, größere sprachliche Einheiten sind vollständige kompositional aus jeweils kleineren Einheiten aufgebaut (vgl. kritisch auch Ziková 2004: 189).

(ii) Es besteht kein grundlegender Unterschied zwischen Wurzeln und Affixen. In beiden Fällen haben wir es mit Paarungen von Laut und Bedeutung zu tun. Sekundäre Unterschiede ergeben sich lediglich aus der Art der Bedeutung (grammatische vs. lexikalische Bedeutung) und der Art der Integration in größere Einheiten (freie vs. gebundene Morpheme), die zu einer Feinklassifizierung der Morpheme einer Sprache herangezogen werden können.

(iii) Die Kombination von Morphemen zu größeren Einheiten unterhalb der Wortebene ist qualitativ nicht verschieden von der Kombination von Morphemen zu Einheiten oberhalb der Wortebene. In beiden Fällen werden Morpheme zu größeren Einheiten zusammengefügt. Mit anderen Worten: Der Unterschied, den die traditionelle Grammatik zwischen Morphologie und Syntax macht, ist nichtig.

Jeder einzelne der hier summierten Punkte muss zumindest als strittig, wenn nicht gar (zumindest in der zugespitzten Form, wie hier formuliert) als offensichtlich falsch gelten. Besonders deutlich werden die Mängel der Morphemkonzeption in Bezug auf (i). Da ich in Abschnitt 3 eine Reihe von Beispielen anführen möchte, die auch für den Fremdsprachenunterricht relevant sind, beschränke ich mich hier auf zwei gut bekannte Problemfälle.

(i) Im Deutschen weisen Verben der schwachen Konjugation ein Segment auf, dem sich problemlos die Präteritumsbedeutung zuordnen lässt: (sag)-te. Ein paralleler Segmentierungsversuch schlägt bei starken Verben dagegen fehl: (sang)-

1 In der angloamerikanischen Forschung spricht man in diesem Zusammenhang von einem Modell auf der Grundlage von „Item and Arrangement“, vgl. z. B. die Übersicht in Plungjan (2003: 37-40; 71-78) oder Aronoff/Fudeman 2005 (46-50). 
???. Bloch (1947) sieht sich daher (bei der Analyse englischer Daten, die den Linguisten vor vergleichbare Probleme stellen wie die deutschen) gezwungen, ein Nullmorph zu postulieren: - $\varnothing$,Präteritum'. Gleichzeitig nimmt er an, es gäbe eine Distributionsregel, die dafür sorgt, dass das lexikalische Morphem /sing/ vor - $\varnothing$ durch das Allomorph sang vertreten wird. Die morphematische Analyse von er sang sieht also folgendermaßen aus: /sing/, cantare + /te/ ,Präteritum', wobei /sing/ im genannten Kontext durch sang und /te/ durch $-\varnothing$ vertreten ist. ${ }^{1}$ Schon Nida (1948: 439-40) kritisiert an dieser Lösung, dass hier der Zeichenbegriff ad absurdum geführt wird, da Bedeutung - statt mit einer empirisch feststellbaren Veränderung im Material - mit einem „unsichtbaren“ Element korreliert: Sprachlicher Inhalt bleibt hier ohne sichtbaren Ausdruck, während gleichzeitig vorhandene Ausdrucksformen zu bedeutungsneutraler „Allomorphie“2 erklärt werden. Nidas eigene Lösung, nämlich ein „Replazem“ („replacive morpheme“) für die Veränderung des Stammvokals anzusetzen (/sing/ $+/ \mathrm{a} \leftarrow \mathrm{i} /$ ), ist meiner Meinung nach zwar einleuchtender, sprengt aber den theoretischen Rahmen des Strukturalismus. Ein „Replazem“ lässt sich wohl kaum noch als sprachliches Minimalzeichen mit Ausdrucks- und Inhaltsseite verstehen. Eher handelt es sich hier um eine Transformationsregel im Sinne der generativen Grammatik, die einen Input nach genau definierten Regeln in den gewünschten Output umformt.

Auch weitere mögliche Lösungen des Ablaut-Problems sind wenig überzeugend. So könnte man z. B. ein diskontinuierliches Morphem /s...ng/ postulieren, ${ }^{3}$ in das jeweils verschiedene grammatische Morpheme (/i/ für Präsens, /a/ für Präteritum) eingesetzt werden. Ob man ein „diskontinuierliches Morphem“/s...ng/ als Zeichen im Saussureschen Sinne betrachten könnte, scheint mir allerdings mehr als fraglich. Allen Autoren, die eine solche Lösung präferieren, würde ich (entsprechend der oben zitierten methodischen Richtlinie von de Saussure) empfehlen, kompetente Sprecher des Deutschen darüber zu befragen, welche Bedeutung sie mit der Lautkette [sy]

1 An dieser Stelle wäre es natürlich notwendig, die genauen Regeln für die Selektion des passenden Allomorphs anzugeben. Wenn man bedenkt, dass in der deutschen Grammatik die Allomorphie bei den starken Verben nur ein Fall unter vielen ist, kann man sich vorstellen, wie komplex das entsprechende Regelsystem sein müsste (vgl. das komplexe System bei Bloch 1947 für das im Vergleich zum deutschen morphologisch einfache englische Verb). Laut Bierwisch (1975: 50, Fußnote 1) hat nie jemand den Versuch unternommen, diese Regeln für das Deutsche genau auszuformulieren. Die Behauptung (z. B. Čermák 2001: 122), dass die Realisierung eines Morphems durch verschiedene Allomorphe eine Erscheinung der parole sei, beruht dagegen offenbar auf einem Missverständnis (vgl. Mugdan 1986: 33f.) und würde - nähme man sie ernst - zentrale Bereiche der Morphologie (wie die hier erwähnten Ablautreihen der deutschen Verben oder etwa auch die Pluralbildung, vgl. Studenten $\times{ }^{*}$ Students) von der systematischen Analyse ausschließen.

2 Allomorphe eines einheitlichen Verbmorphems sind die ablautenden Stammformen auch für Čermák (2001: 124), der allerdings im Unterschied zu Bloch keine Erklärung dafür anbietet, wie eine per definitionem bedeutungsneutrale Alternation zwischen Allomorphen zu einer Bedeutungsänderung führen kann.

3 Auch diese Lösung wird in Bloch (1947) erwogen, dann allerdings verworfen. 
assoziieren. Die alternative Lösung, sang als Portemanteau-Morphem ${ }^{1}$ zu betrachten, in dem die Bedeutungskomponenten ,cantare' und ,Präteritum 'vereint sind, verwischt den allseits angenommenen Unterschied zwischen "grammatischen" und „lexikalischen Morphemen“ und stellt zudem eine empfindliche Aufweichung der theoretischen Annahme (i) dar, der zufolge Morpheme die kleinsten Zeichen, demnach also weder auf der Ausdrucks- noch auf der Inhaltsseite (Zeichen = untrennbare Einheit von Form/Inhalt) weiter segmentierbar sind.

Den umgekehrten Fall einer Diskrepanz zwischen der Gliederung auf der Inhalts- und Ausdrucksseite des sprachlichen Zeichens, d. h. einen „Überschuss“ von segmentalem Material gegenüber einem „Überschuss" von Inhalt im vorher diskutiertem Fall (vgl. Matthews 1972: 77), stellt etwa das deutsche InfinitivFormativ $z u$ dar. Nachdem man z. B. in dem Satz Er fängt an zu singen alle interpretierbaren Inhaltskomponenten den entsprechenden Lautketten auf der Ausdrucksseite zugeordnet hat $\left(/ \mathrm{e}^{\mathrm{a}} /=3\right.$. Pers. Sg. m., $/ \mathrm{fg} \mathrm{gk} . . . \mathrm{an} /=$,incipere', $/ \mathrm{t} /=3$. Pers. Sg. Präs., /sin/ = , cantare', /ən/ = Infinitiv), bleibt für die Lautkette $z u$ keine Inhaltskomponente mehr übrig. ${ }^{2}$ Damit würde $z u$ das Kriterium, nach dem Morpheme definiert sind, nicht erfüllen. Der oben betrachtete Satz würde demzufolge den Präzedenzfall einer nicht exhaustiv in Morpheme zerlegbaren sprachlichen Struktur darstellen. ${ }^{3}$ Die Behauptung, $z u$ habe zwar keine Bedeutung, im Sinne der oben zitierten Definition von Čermák (2011) aber eine (rein sprachimmanente) Funktion (z. B. die Funktion, den Infinitiv in die Satzstruktur zu integrieren), hilft hier auch nicht weiter, da es keine unabhängige Basis dafür gibt, diese Behauptung zu belegen. Wir würden uns viel mehr in einen logischen Zirkel begeben, da die Funktion von $z u$ durch dessen obligatorische Anwesenheit und die obligatorische Anwesenheit von $z u$ wiederum durch dessen vorgebliche Funktion gerechtfertigt würde. Andere Sprachen (z. B. das Tschechische: Začiná zpivat) belegen darüber hinaus, dass es in natürlichen Sprachen keineswegs notwendig ist, einen Infinitiv durch ein spezielles „Morphem“ in den Satz einzugliedern, weswegen auch kein prinzipieller Bedarf an Elementen besteht, die eine solche „Funktion“ übernehmen würden. Auch im Rahmen eines funktional ausgerichteten Sprachmodells wird man also nicht umhin kommen einzuräumen, dass nicht jedes Element, das an der Oberfläche erscheint, gleichzeitig auch eine Funktion hat (vgl. Welke 2002: 6f.). Dies untergräbt aber wiederum die Grundannahme des strukturalistischen Modells, dass

1 Der Begriff stammt von (Hockett 1947), allerdings nicht die hier polemisch vorgeschlagene Anwendung auf durch Ablaut gebildete Stammformen.

2 Vgl. auch die Diskussion in Chomsky $(1957, \$ 9.2 .7)$ zu to im Englischen. Chomsky spricht hier von einem „Morphem“, denen keine Bedeutung zugeordnet ist!

3 Das Postulat, dass jede sprachliche Struktur vollständig in Morpheme zerlegbar ist und daher jedes Segment einem Morphem zugeordnet werden muss, ist in Bloomfield (1933) formuliert. Andere Konzeptionen (z. B. Hockett 1947) lassen dagegen auch „leere Morphe“ zu (vgl. auch die Diskussion in Aronoff/Fudeman 2005: 82f.). Die mit einer solchen Konzeption verbundenen Probleme können hier nicht weiter erörtert werden. 
nämlich sprachliche Ketten als Zeichen, also als lückenlose Aneinanderreihungen von doppelseitigen Minimalzeichen (Morphemen) aufzufassen sind.

Hinsichtlich Punkt (ii), in dem die grundsätzliche Wesensgleichheit von Morphemen aller möglichen Subtypen (reflektiert durch die Einführung des Oberbegriffes „Morphem") ${ }^{1}$ behauptet wird, sei darauf hingewiesen, dass sich gerade auch im Deutschen Stämme und Affixe grundsätzlich verschieden verhalten. Dies zeigt sich schon in der phonemischen Realisierung dieser Elemente, die bei Affixen und Stämmen verschieden ist: Während Stämme Zugang zum gesamten Phonemrepertoire des Deutschen haben, sind Flexionssuffixe und tendenziell auch gebundene Präfixe (be-, er-, ver-, zer-) auf den Schwa-Vokal beschränkt (vgl. die Unterscheidung von wortwertigen Morphemen und nicht wortwertigen Morphemen bei Nübling et al. 2010: 18). Solchen Unterschieden kann man selbstverständlich dadurch gerecht werden, dass man zwischen "Stammmorphemen“ und „Flexionsmorphemen“ (Thieroff/Vogel 2009: 1) unterscheidet; welchen Vorteil die mit Hilfe der Derivationsbasis "-morphem“ gebildeten Termini gegenüber denjenigen ohne diese Basis haben sollen, bleibt dabei im Dunklen. Wie dem auch sei, man wird zumindest zugestehen müssen, dass nur "Stammmorpheme“ außerhalb eines größeren Kontextes mit Bedeutungen assoziierbar sind. Isolierte "Flexionsfixmorpheme“ (etwa das durch den dentalen Verschlusslaut gebildete Morphem / $\mathrm{t} / \mathrm{im}$ Deutschen) evozieren selbst bei den kompetentesten Sprechern einer Sprache keinerlei begriffliche Vorstellungen.

(iii) Durch die Einführung des Begriffs „Morphem“ wird der in der traditionellen Grammatik zentrale Begriff, „Wort" aus der Grammatiktheorie eliminiert, denn wenn die Zuordnung von Laut und Bedeutung bereits unterhalb der Wortebene einsetzt und größere Strukturen sich regelmäßig durch das Aneinanderreihen von kleineren Bausteinen aufbauen lassen, besteht für die Bezugnahme auf die Einheit „Wort" kein Grund mehr. Wörter sind evtl. noch als „linguistische Folklore“ akzeptabel, für die konsistente Analyse von sprachlichen Strukturen sind sie dagegen überflüssig. Folgerichtig beschreiben die amerikanischen Strukturalisten auch Sätze als Ketten von Morphemen, vgl. Bloch (1947: 398f.): „Any sentence [!], phrase, or complex word can be described as consisting of such-and-such morphemes in suchand-such an order". Wort und Morphem sind also, was die Wahl der Grundeinheit der Grammatik betrifft, konkurrierende Begriffe (Römer 2006: 4). Es ist hier begreiflicherweise nicht der Ort, die Frage nach der Grundeinheit der menschlichen Sprache zu beantworten. Es soll allerdings vehement auf die oft verschleierte Tatsache hingewiesen werden, dass ein Modell, das aufder Grundlage von Morphemen operiert, prinzipiell unvereinbar ist mit der Formenlehre der traditionellen Grammatik, die

$\overline{1}$ Einige Linguisten (z. B. Tomáš Káňa, mündl. Mitteilung) sehen die Nützlichkeit des Morphembegriffes gerade darin, Wurzeln, Stämme und Affixe terminologisch zu einer Kategorie zusammenzufassen. Wozu ein solcher Oberbegriff gut sein soll, da es doch (meines Wissens) keine grammatischen Regeln gibt, die gleichermaßen auf Wurzel, Stämme und Affixe Bezug nehmen, bleibt dabei unklar. 
auf der Vorstellung von (lexikalischen) Wörtern und deren Paradigmen aufbaut, eine Vorstellung, wie sie allgemein auch im Fremdsprachenunterricht vorausgesetzt wird.

Die Schwierigkeiten, die sich aus der Analyse von Sprachmaterial mit Hilfe des Morphembegriffes ergeben, hat dazu geführt, dass heute in der Linguistik vielfach die Gegenposition zu (i)-(iii) vertreten wird. Insbesondere gehen viele linguistische Schulen oder einzelne Forscher von (a)-(c) aus:

(a) Die Zuordnung von Form und Inhalt erfolgt in menschlichen Sprachen indirekt. Sie ergibt sich aus der Gesamtsicht der Grammatik und braucht nicht bei jedem einzelnen Element, das sich an der Oberfläche aufgrund formaler Prozeduren ermitteln lässt, vorzuliegen (Ruwet 1967: 206f.). Daher ist nicht jedes Formelement gleichzeitig auch ein zweiseitiges sprachliches Zeichen.

(b) Als Minimalzeichen im Saussureschen Sinne kommt allenfalls das Wort in Frage (Anderson 1992: 49; Wurzel 2000: 12). Kleinere Einheiten (wie etwa Flexionssuffixe u. ä.) sind für sich genommen keine sprachlichen Zeichen und werden erst im Rahmen des Wortes signifikant.

(c) Neben der Addition von sprachlichem Material zum Ausdruck von Bedeutung (das einzig mögliche Verfahren im Rahmen eins strengen Item-und-Arrangement-Modells) muss auch die Modifikation oder die Tilgung vorhandenen Materials als bedeutungssignifikant anerkannt werden. Modifikationen und Tilgungen können jedoch nicht als zweiseitige sprachliche Zeichen verstanden werden, sondern nur als Operationen über solchen Zeichen. Als Operationen sind Modifikation und Tilgung eine Alternative zur Markierung von grammatischen Bedeutungen durch Morpheme (vgl. z. B. Wurzel 2000; Janakiev 2010). Eine Extremposition stellt z. B. der Ansatz von Anderson (1982; 1992) dar, der alle Markierungstypen (also auch die segmentalen) auf Operationen über Minimalzeichen (d. h. Wörtern) zurückführt. ${ }^{1}$

Damit ergibt sich am Anfang des 21. Jahrhunderts in verwirrend buntes Bild. Neben Autoren, die den Begriff „Morphem“ in der ursprünglichen, strukturalistischen Bedeutung im Sinne von „doppelseitigem Minimalzeichen“ verwenden (Čermák

1 Die grundsätzliche Unterscheidung, die Wurzel (2000) zwischen Prozessen, die nur aufgrund des Kontrasts der modifizierten Form zur Ausgangsform signifikant sind, und Morphemen, die unabhängig vom Kontrast zu einer Ausgangsform Bedeutungen tragen, trifft, scheint mir zu kurz gegriffen. Auch das Auftreten von segmentalem Material ist nur dann signifikant, wenn es neben der Form, die das fragliche Material enthält, eine andere Form gibt, die dieses Material nicht enthält. So entsteht die pluralisierende Wirkung des Suffixes -(e) $n$ in der Wortform Leichen nur dadurch, dass es eine andere Wortform (nämlich Leiche) gibt, die dieses Suffix nicht enthält. Dies lässt sich dadurch belegen, dass das in materieller Hinsicht identische Element -en in der Wortform Zeichen keine pluralisierende Wirkung hat, da eine entsprechende Kontrastform $\left({ }^{*} Z e i c h,{ }^{*} Z e i c h e\right)$ fehlt. In beiden Fällen (der Modifikation und der Hinzufügung von Material) beruht der signifikative Effekt also auf demselben Prinzip. 
2001; 2011), gibt es eine ganze Reihe von anderen Autoren und Konzeptionen, die den Morphembegriff explizit ablehnen (Matthews 1972; Bierwisch 1975; Anderson 1982; 1992). In vielen weiteren Konzeptionen spielt der Morphembegriff - auch wenn die entsprechenden Autoren sich nicht explizit dazu äußern - weder terminologisch noch konzeptionell eine Rolle (Stump 1991; Eisenberg 2006; Hudson 2007).

Noch prekärer wird die terminologische Situation dadurch, dass viele Autoren zwar den Begriff, Morphem“verwenden, damit allerdings völlig andere Vorstellungen als die (mitteleuropäischen oder amerikanischen) Strukturalisten verbinden: So sind etwa für Vendryès (1921) und die romanistische Tradition (z. B. Clément 1996) all diejenigen sprachlichen Mittel „Morpheme“, die grammatische (im Unterschied zu lexikalischen) Informationen kodieren, inkl. Intonation und Wortstellung (!). ${ }^{1}$ Die Autoren im Umfeld der „Natürlichen Morphologie“ (z. B. Wurzel 2000) sowie an Wurzel anknüpfende Arbeiten (etwa Bittner 1996; Janakiev 2010) betrachten grammatische Morpheme dagegen nur als Spezialfall der Markierung von grammatischer Information am Wort, der immer dann vorliegt, wenn keine Prozesse (Operationen am Wortkörper), sondern segmentales Material zum Einsatz kommt. Das Präteritum der schwachen Verben wird nach dieser Auffassung also durch ein „Morphem“ markiert (/te/ z. B. in sagte), das Präteritum der starken Verben (z. B. singen $\rightarrow$ sang) hingegen nicht, da kein segmentales Material hinzugefügt wird. Daneben verwenden viele generativistische Autoren den Begriff „Morphem“ (sofern sie ihn überhaupt verwenden) gleichbedeutend mit „morphosyntaktische Merkmale“, unabhängig davon, ob diesen Merkmalen eine spezifische, materielle Realisierung zukommt oder nicht (so z. B. Chomsky 1957; ${ }^{2}$ Ruwet 1967; Halle/Marantz 1993). ${ }^{3}$ Andere wiederum (z. B. Aronoff 1976; Bierwisch 1989) bezeichnen auch bedeutungsleere segmentale Einheiten als Morpheme, soweit grammatische Regeln

1 Im selben Sinne (d. h. unter Einbezug der Wortstellung) benutzt schon Trnka (1932) den Begriff „Marker“.

2 Die Verwendung des Begriffs „Morphem“ bei Chomsky (1957) scheint mir insgesamt inkonsistent zu sein. Einerseits ( $\$ 4.3$, zit. nach der fr. Übersetzung, $S$. 35f.) heißt es, eine Grammatik müsse die korrekte Morphemabfolge generieren, die in einem zweiten Schritt mit der passenden phonologischen Form zu versehen sei. Da ein möglicher terminaler Knoten der Grammatik Past ist (Bsp. 19 vi), lässt sich diese Passage nur so verstehen, dass ein „Morphem“ ein Bündel morphosyntaktischer (evtl. auch lexikalischer) Merkmale ohne Ausdrucksseite ist. An anderer Stelle (z. B. \$ 9.4., zit. nach der fr. Übersetzung, S. 117) heißt es dagegen wieder, dass man für eine umfassende Analyse einer Sprache auch die Bedeutung von „Wörtern und Morphemen“ kennen muss. Hier kann mit „Morphem“ also nur die Ausdrucksseite einer sprachlichen Einheit ohne deren Inhaltsseite gemeint sein. Diese Inkonsistenz rührt natürlich daher, dass für das rein formale System in Chomsky (1957) der bilaterale Morphembegriff nicht die geringste Rolle spielt.

3 Aus diesem Verständnis ergeben sich solche, für Vertreter des strukturalistischen Morphembegriffes paradoxe Formulierungen, wie ,das Morph (= phonologisches Material) wird in ein Morphem (= terminaler Knoten, der nach best. morphosyntaktischen Merkmalen spezifiziert ist) eingesetzt ${ }^{\text {‘ }}$ (vgl. etwa die kritische Zusammenfassung in Stump2007: 40). 
auf sie Bezug nehmen. ${ }^{1}$ Von einer einheitlichen, in der modernen Sprachwissenschaft allgemein akzeptierten Terminologie kann also keine Rede sein. ${ }^{2}$

Ziel des in diesem Abschnitt zusammengestellten Überblicks ist es nicht, die linguistische Brauchbarkeit des strukturalistischen Morphembegriffes generell in Frage zu stellen. Zu dieser Frage wird sich jeder Leser seine eigene Meinung gebildet haben. Was allerdings sehr wohl in Frage gestellt werden soll, ist die Annahme, der Begriff „Morphem“ sei so, wie er in den Lexika und einführenden Skripten häufig definiert wird, allgemein anerkannte Grundlage der linguistischen Forschung am Anfang des 21. Jahrhunderts. Im nächsten Abschnitt soll geprüft werden, ob der Morphembegriff im Sinne von Bußmann (1990) oder Čermák (2011) Studierenden in DaF-Studiengängen außerhalb des deutschsprachigen Raumes bei der Analyse fremdsprachlicher Strukturen hilft bzw. für das Verständnis des grammatischen Systems des Deutschen (soweit es für zukünftige Fremdsprachenlehrer an Grundschulen erforderlich ist) notwendig ist.

\section{Nützlichkeit für das Verständnis fremdsprachlicher Strukturen}

Anderson (1992:51) leitet seine Diskussion des strukturalistischen Morphembegriffes mit einer Beobachtung aus dem pädagogischen Alltag eines Hochschuldozenten ein, der mit der Durchführung von Einführungskursen in die Linguistik betraut ist:

The intuitive appeal of the structuralist morpheme is not hard to see, of course. Any linguist who has had the task of explaining morphological structure to an introductory class has probably had the experience that after seeing only a small number of examples of words analyzed into morphemic constituents, most students can readily extend this analysis to new words, even in unfamiliar languages. Of course, the cases that are readily comprehended are those in which the morphological structure fits naturally with the assumptions above [...] Other, more problematic structural types are much more difficult for students to accommodate to this picture - as indeed they were for the structuralist theoreticians of the 1940s and 1950s. (Anderson 1992: 51)

Schenkt man den gängigen Skripten zu Einführungskursen in die Sprachwissenschaft Glauben, dann handelt es sich bei den im Zitat erwähnten „problematischeren Strukturtypen“ um ein unbedeutendes Randphänomen. Das Paradebeispiel eines solchen „problematischeren Strukturtyps“ wäre etwa das vielfach genannte Kompositum Himbeere, welches (wegen fehlendem signifié beim Erstglied) nicht

1 In älteren generativistischen Arbeiten (Chomsky 1965: 16; Ruwet 1967: 207; 343f.) wurde der Versuch unternommen, den Begriff „Morphem“ in der Bedeutung, segmentales Material" durch den neutralen Begriff „Formativ“ zu ersetzen. Soweit ich sehen kann, hat sich diese terminologische Innovation (die eigentlich an den Sprachgebrauch der Junggrammatiker anknüpft, vgl. etwa „Formans“ bei Leskien 1910) nicht allgemein durchgesetzt.

2 Zur Vieldeutigkeit des Begriffes „Morphem“ bereits Mitte der 80er Jahre, vgl. Mugdan (1986). 
reibungslos segmentierbar ist (vgl. z. B. Ernst 2004: 109; Šimečková 2004: 78; Römer 2006: 28; Geislerová 2007: 10). Der Eindruck, dass Fälle wie Himbeere die Ausnahme darstellen, trügt jedoch; ganz im Gegenteil handelt es sich hier um den strukturellen Normalfall, wovon sich jeder problemlos selbst überzeugen kann, indem er versucht, Sprachmaterial aus beliebig zusammengesuchten Sätzen nach strukturalistischen Grundsätzen in Morpheme (also in Minimalzeichen aus Ausdrucks- und Inhaltsseite) zu zerlegen. Im Folgenden will ich einige, völlig unauffällige Beispiele aus dem Deutschen anführen und zeigen, vor welche Schwierigkeiten eine simple Aufgabenstellung wie „Zerlegen Sie folgende Strukturen in ihre Morpheme" Studierende in DaF-Studiengängen stellen könnte.

(a) In der Partizipialform gelacht lassen sich (durch Vergleich mit hunderten von weiteren Partizipialformen) drei Elemente segmentieren: ge-lach-t. Bei -lachhandelt es sich offenbar um ein „lexikalisches Morphem“, da dem sprachlichen Ausdruck hier auf der Inhaltsseite eine relativ klar fassbare lexikalische Bedeutung gegenübersteht. Wie aber steht es mit den beiden Segmenten ge- und -t? Lassen sich diesen Ausdruckselementen Inhalte zuordnen? Gesetzt den Fall, wir akzeptieren das Merkmal ,Perfekt' (oder wahlweise, Partizip') als zulässigen, sprachlichen Inhalt (was keineswegs unproblematisch ist); muss dieses Merkmal nun dem Element geoder dem Element $-t$ zugeordnet werden? Die Existenz von anderen Partizipien wie belach-t, bei denen das Element ge- fehlt, zwingt offenbar zur zweiten Lösung. Welcher Inhalt kann aber dann dem verbleibenden Element ge- zugeordnet werden? Offenbar haben wir es mit einem bedeutungs- und funktionslosen Morph zu tun, das dennoch obligatorisch bei gewissen lexikalischen Morphemen - zusammen mit dem Partizip-Morphem - erscheinen muss (vgl. * Sie hat lacht). Wenn jedoch Partizipialformen wie *lacht ohne ge- ungrammatisch sind, müsste man daraus eigentlich weiter schließen, dass ge- eine weitere, obligatorische Ausdrucksform für ,Partizip' ist. Derselbe Inhalt wird also an zwei verschiedenen Stellen ausgedrückt. In der Fachliteratur spricht man hier von "erweiterter Exponens" (vgl. Aronoff/ Fudeman 2005: 152-155, die den Begriff von Matthews 1972: 82 übernehmen) ${ }^{1}$ und betrachtet die Existenz solcher Fälle als Beleg dafür, dass die Zuordnung von Form und Bedeutung in natürlichen Sprachen eben genau nicht eins-zu-eins erfolgt. $^{2}$

1 Die häufig angebotene Lösung, ge- und -t als „Zirkumfix“ zu betrachten (z. B. Geislerová 2007: 12; Heringer 2009: 31; Thieroff/Vogel 2009 :7), widerspricht dem Geist des Morphem-Modells, da es sich hier um kein Minimalzeichen handelt: Die Ausdrucksseite ist nämlich weiter segmentierbar, was sich durch die Unterbrechbarkeit der Lautkette ge-t durch lexikalisches Material und das separate Vorkommen von -en/-t belegen lässt. Vgl. die Definition von „Zirkumfix“ bei Čermák (2011: 17): „Cirkumfix [...] není jeden afix, ale diskrétní kombinace několika afixů s pevným významem nebo funkcí $[\ldots]$ “. Der Autor zieht aus dem Gesagten allerdings nicht die logisch zwingende Schlussfolgerung, dass es sich dann bei Zirkumfixen um keine Minimalzeichen und damit auch um keine Morpheme handelt.

2 Weitere Probleme mit dem Präfix ge- seien hier nur kurz angedeutet: (i) Ist be- ein Allomorph desselben Morphems /ge/, da ge- und be- in gelacht und belacht in komplementärer Distribution 
(b) Wie wären Verben wie telefonieren, tapezieren oder massieren zu segmentieren? Insbesondere: Handelt es sich bei dem durch den Vergleich der Formen isolierbaren Segment -ier- um ein Morphem? Komárek (2006: 101) setzt hier für analoge Elemente im Tschechischen eine eigene Klasse von morphematischen Einheiten an, die er mit dem Begriff „Konnektem“ bezeichnet. Welcher Inhalt wäre aber einem Morphem (Konnektem) -ier- zuzuordnen? Offenbar keiner, da weder eine begriffliche Bedeutung noch eine rein grammatische Funktion (z. B. die Funktion, Verbalstämme zu kennzeichnen) vorliegt. Letztere Behauptung lässt sich durch die Existenz zahlloser Verbalstämme ohne das Suffix-ier- belegen. Auch die Vermutung, -ier- könne als Ableitungsmorphem (evtl. besonders für die Adaption von Fremdwörtern) betrachtet werden (Fleischer/Barz 2007: 311) ist nicht stichhaltig, da (i) Fremdwörter auch ohne -ier-adaptiert werden können (vgl. mailen, scannen, duschen), (ii) in vielen Fällen keine mögliche Derivationsbasis existiert, von der ier- entsprechende Verben ableiten könnte (z. B. rasieren $<{ }^{*}$ Ras, passieren $<\neq$ Pass, grassieren $<{ }^{*}$ Grass). Ähnliche Probleme entstehen für eine morphematische Analyse in vielen Sprachen, die über Themenvokale beim Verbstamm verfügen. Eine überzeugende Lösung für dieses Problem ist mir nicht bekannt.

(c) Müssen wir (wegen der formalen Identifizierbarkeit) für die Wortformen Zeichen, Kissen, Wappen ein Morphem /en/ ansetzen? Zwar lässt sich auch hier kein begrifflicher Inhalt identifizieren, der die zweite Seite eines Minimalzeichens bilden könnte, aber wenn wir im Falle von -ier- eine Stammbildungs- bzw. Ableitungsfunktion als hinreichend für die Rechtfertigung eines Morphems betrachten, müssten wir auch im Falle von -en dieses Argument akzeptieren. Čermák (2011:17) unterscheidet $^{1}$ bei einem analogen Fall im Tschechischen zwischen segmentierbaren Formen wie květen ,Mai` (weil eine offensichtliche Ableitungsbeziehung zu dem Wort květ ,Blüte‘ besteht) und nicht segmentierbaren Formen wie buben ,Trommel, breben ,Kamm', červen ,Juni' (da die hypothetischen Derivationsbasen entweder nicht existieren oder eine andere Bedeutung als die entsprechenden Derivate haben). Dementsprechend müsste man bei den deutschen Beispielen oben das aus zwei „Morphemen“ zusammengesetzte Zeich-en (Zeich-als Allomorph zu /zeig/) von den anderen, jeweils nur ein Morphem umfassenden Formen trennen. Ob eine solche Trennung - sowohl für das Deutsche als auch für das Tschechische - sinnvoll wäre, wage ich allerdings zu bezweifeln. Aus morphologischer Sicht verhält sich das Element -en in

stehen (sofern man die Akkusativ-Ergänzung als syntaktische Umgebung einbezieht)? (ii) Ist ge- in gelacht und Gelächter dasselbe Morphem? (iii) Ist ge- dann auch in Gelächter und Geländer dasselbe Morphem (auch wenn Geländer keine verbale Derivationsbasis hat)? (iv) Muss man für Sie hat nicht kommen können bei können ein Null-Allomorph des Morphems /ge/ ansetzen?

1 Gleichzeitig merkt er an, dass entsprechende Entscheidungen in viele Fällen nicht systematisch zu rechtfertigen und daher willkürlich sind. An diese Feststellung müsste sich nun eigentlich die Frage anschließen, in wieweit ein theoretischer Rahmen den sprachlichen Daten angemessen ist, der den Analysten in großem Umfang zu willkürlichen, durch nichts zu rechtfertigenden Entscheidungen zwingt. 
allen Fällen gleich (vgl. Zeichen ${ }_{\mathrm{pl},}$, Kissen ${ }_{\mathrm{pl}} \times$ Kinder $_{\mathrm{pl}}$, Kreuze $_{\mathrm{pl}}$; prvního květnal června, $v$ květnulv červnu, květnovýlč́rvnový) und muss daher für die entsprechende Regel als eigenständiges Element identifizierbar sein. Für das Deutsche spricht Nübling (2008) von einem „Pseudo-Suffix“. „Pseudo-Suffixe“ sind aber (wegen dem Fehlen der Inhaltsseite) keine sprachlichen Zeichen und damit keine Morpheme.

(d) Die Formen Kinder, Kreuze oder Türen weisen eine sichtbare Pluralmarkierung auf. Nun stellt sich die Frage: Handelt es sich bei den entsprechenden Markern (-er, $-e,-e n)$ um Allomorphe desselben Pluralmorphems? Oder repräsentieren die Marker, aufgrund der deutlichen Unterschiede in der Ausdrucksform, trotz Inhaltsgleichheit verschiedene Morpheme? Man sollte meinen, dass ein derart elementarer Fall in der Morphologie des Deutschen schon lange eindeutig geklärt ist. Dem ist nicht so. Zwar bewerten wohl die meisten Autoren die Variation in der deutschen Pluralmarkierung (analog zu Nida 1948 für das Englische) als Allomorphie (z. B. Bußmann 1990: 52; Nüblimg 2008); es gibt aber auch namhafte Abweichler, wie etwa Heringer (2009: 28), der meint, die Zusammenfassung phonologisch nicht aufeinander beziehbarer Formen zu einem Morphem „[...] wirkt etwas gekünstelt“. Wenn sich schon die Fachleute nicht einig sind, wie soll man hier dann fremdsprachigen Studierenden in DaF-Studiengängen eine Entscheidung abverlangen, ließe sich fragen.

(e) Präfixe sind häufig mit bestimmten Bedeutungen assoziierbar. Das Präfix ent-, wie etwa in entfernen, entnehmen oder entfesseln, drückt die räumliche Separierung eines Objekts von einer Grundfigur aus. ent- müsste in den genannten Beispielen daher als Morphem betrachtet werden. Liegt dann auch in empfehlen ein Allomorph desselben Morphems /ent/ vor? Wenn ja, ist -fehlen in empfehlen dann dasselbe Morphem wie in fehlen oder befehlen? Sollte es sich in allen drei Fällen um dasselbe Morphem handeln, wäre weiter zu klären, wieso im Präteritum jeweils andere Allomorphe eintreten (vgl. er fehl-te $\times$ er emp-fahl). Entschließt man sich angesichts dieser Fakten, zwei verschiedene Morpheme für fehlen anzusetzen (/fehl ${ }_{1}$ für fehlen, /fehl / für emp-fehlen), kommt man in Erklärungsnot, wenn man die Inhaltsseite von / fehl 2 / bestimmen soll. Offenbar hat -fehl-außerhalb von empfehlen gar keine Bedeutung. Mir scheint, dass eine Analyse, der die in Abschnitt 1 zitierten Morphemdefinitionen zugrunde liegen, hier nur zu dem Schluss kommen kann, dass die Form empfehl- (wie auch die Form befehl-) aus nur einem Morphem besteht, da keine der hypothetisch identifizierbaren Untereinheiten in Isolation eine eigenständige Bedeutung hat. Die morphematische Analyse gerät damit in Widerspruch zu einer rein formalen Analyse, die Aufgrund des Vergleichs mit den Formen entnehmen, entfesseln und empfinden einerseits, und fehlen, befehlen oder verfehlen andererseits die Segmentation in Präfix und Stamm (emp-fehlen) ermöglicht. Studierende von Deutsch als Fremdsprache, die zunächst die formale Seite der zu erwerbenden Wortformen bewältigen müssen, werden durch Überlegungen wie die eben angestellten vermutlich eher verunsichert, als dass sie einen Gewinn daraus ziehen könnten. 
(f) Ähnliche Probleme wie sie bei empfehlen in (e) sind im Bereich der Präfigierung Legion. Wie wäre etwa das Substantiv Grünanlage morphematisch zu analysieren? Als Grün-an-lag-e? Ist -lag- hier - trotz völlig anderer Bedeutung - ein Allomorph von /lieg/? Und welche Bedeutung käme dem hypothetischen Morphem /an/ zu? Oder sollte man Besitz als Be-sitz segmentieren und die zweite Komponente dem Morphem /sitz/ von sitzen zuordnen? Das entspräche wohl nicht mehr dem heutigen Stand des lexikalischen Systems des Deutschen. Ein Basismorphem /besitz/ wiederum würde die eindeutig gegebenen formalen Bezüge zu Benennung, Beziehung, Bezahlung einerseits und zu Sitz, Wohnsitz, Vorsitz andererseits vernachlässigen.

(g) Analoge Probleme ergeben sich bei der morphematischen Analyse von Suffixen. Folgt man der Definition von „Morphem“ als doppelseitigem Zeichen, dann müssen die Adjektiv freund-lich, dümm-lich, lächer-lich, läst-ig wie angegeben segmentiert werden, da sie die „Basismorpheme“/freund/, /dumm/, /lach/ und /last/ enthalten; herrlich oder lustig kann dagegen nicht segmentiert werden, da sich die erste Komponente nicht den Morphemen /herr/ bzw. /lust/ zuordnen lässt: die herrliche Natur in Südmähren hat nichts mit ,Herren' zu tun, ein lustiger Film nichts mit ,Lust' (auch wenn das Ansehen eines solchen Films u. a. auch als lustvoll empfunden werden kann).

(h) Die gängigen Skripten und Einführungskurse in die Sprachwissenschaft unterscheiden zwischen "grammatischen" und „lexikalischen" Morphemen (z. B. Ernst 2004: 108). Die Klassifizierung erfolgt aufgrund der Bedeutung, wobei grammatische Bedeutungen, die sich zu grammatischen Kategorien zusammenfassen lassen, ${ }^{1}$ von lexikalischen Bedeutungen, die unter keine grammatische Kategorie fallen, zu unterscheiden sind. Dieses System lässt sich relativ reibungslos auf Fälle wie ich suchte anwenden, wo sich /ich/ und /te/ (aufgrund der exhaustiven Zugehörigkeit zu den Kategorien ,1. Pers. Sg.' bzw. ,Präteritum') als „grammatische Morpheme“ und /such/ (wegen der fehlenden Zugehörigkeit zu einer grammatischen Kategorie) als „lexikalisches Morphem“ identifizieren lassen. Wie steht es aber z. B. mit der Konjunktion wenn? Entsprechend der traditionellen Grammatik wäre es wünschenswert, Konjunktionen und andere „Strukturwörter“ dem grammatischen Bereich zuordnen zu können. Leider stellt die traditionelle Grammatik aber keine grammatische Kategorie bereit, in der sich eine Konjunktion wie wenn einordnen ließe. Ohne tiefgreifende Revision des Kategoriensystems ${ }^{2}$ müsste man demnach wenn als „lexikalisches Morphem“ klassifizieren.

1 Vgl. z. B. die Definition von „grammatischer Kategorie“ bei Plungjan (2003: 109). Demnach sind grammatische Kategorien Mengen von sich syntagmatisch ausschließenden abstrakten Bedeutungen, von denen immer eine am nach der jeweiligen Kategorie spezifizierbaren Trägerwort obligatorisch realisiert werden muss.

2 Natürlich fehlt es nicht an entsprechenden Vorschlägen, vgl. die Kategorie „Taxis“ in der russischen funktionalen Grammatik (z. B. Bondarko 1984). Für den DaF-Unterricht sind solche Vorschläge aber wohl kaum geeignet. 
(i) Betrachten wir zum Abschluss noch die Probleme, die sich bei einer Klassifizierung in ,freie“ und,gebundene Morpheme“ergeben. Das oben besprochene Präfix ent-ist offenbar ein gebundenes Morphem. Ist Haus dann ein freies Morphem? Folgt man der Argumentation zum Verbstamm tanz- in Römer (2006: 28), muss die Antwort - wider Erwarten und entgegen einer weit verbreiteten Ansicht (vgl. z. B. Ernst 2004: 109) - nein lauten. Römer argumentiert, dass das Morphem /tanz/ als gebundenes Morphem interpretiert werden muss, da die frei vorkommende Form tanz! (im Imperativ) morphematisch eine Verkettung von Stammmorphem und Nullmorphem für den Imperativ darstelle: $\tan z-\varnothing$. $\tan z-$ kommt somit nur in Verbindung mit anderen, grammatischen Morphemen vor und muss als gebunden gelten. In diesem Fall muss die Form Haus vermutlich analog als Verkettung von Stammmorphem und Nullmorph für ,Nominativ' bzw. ,Akkusativ' analysiert und die Form Haus morphematisch als /Haus/+/Ø/, d. h. als obligatorische Verknüpfung von lexikalischem und grammatischem Morphem betrachtet werden. Hier liegt nicht nur ein eklatanter Verstoß gegen den gesunden Menschenverstand vor, der eher an praktischen Fragen interessierten Lehramtsstudenten an pädagogischen Fakultäten in Tschechien kaum zu vermitteln ist, sondern auch eine Missachtung des morphologischen Typs des Deutschen als einer Sprache, die tendenziell zum Typus der Sprachen mit Grundformflexion gehört (Eisenberg 2006: 153).

(j) Die Artikelform dem stellt offensichtlich ein freies Morphem ${ }^{1}$ dar. Nun ergibt sich allerdings die Frage, wie - $m$ in beim Arzt zu analysieren ist. Da bei dem Arzt (bei dem ich seit Jahren in Behandlung bin) und beim Arzt im Allgemeinen als Varianten aufgefasst werden, wäre - $m$ hier als Allomorph von dem aufzufassen. Das Morphem /dem/ hätte somit zwei Allomorphe, von denen das eine als freies Morph, das andere als gebundenes Morph vorkommt. Solange man nicht festlegt, welches dieser beiden Allomorphe als Grundalternante zu betrachteten ist (und nach welchen Regeln dies zu geschehen hat), lässt sich das Morphem /dem/ also weder als freies, noch als gebundenes Morphem klassifizieren.

Die oben angeführten Beispiele, die sich nahezu beliebig vermehren ließen, haben wohl eines gezeigt: Die Anwendung der Definition von „Morphem“ als „kleinster bedeutungstragender Einheit der Sprache“ auf konkretes Sprachmaterial stellt Studierende, die Deutsch als Fremdsprache erwerben, (und nicht nur diese!) vor ganz erhebliche Probleme, ohne dass der Gewinn, den sie aus der Beschäftigung mit Fragen der Segmentierbarkeit und Zuordnung der Segmente zu Morphemen ziehen könnten, ersichtlich wäre. Aus Sicht von Studierenden ist bei Aufgabenstellungen, die die Anwendung des Morphembegriffes verlangen, die günstigste Strategie vermutlich die, jedes formal erkennbare Affix sowie die nach Abtrennung der Affixe übrig bleibenden Stämme als „Morpheme“ zu bezeichnen. In diesem Fall hätten die Studierenden v. a. gelernt, dass wissenschaftliche Definitionen auswendig zu

1 Der Frage, ob man den Artikel morphematisch nicht weiter segmentieren müsste, gehe ich hier lieber nicht nach. 
lernende und bei Prüfungen zu reproduzierende Merksätze sind, die bei der Lösung von konkreten Problemen aber unbedingt vernachlässigt werden müssen, um zu brauchbaren Ergebnissen zu kommen. Die Festigung einer Haltung, aus der heraus theoretische Inhalte als unverständlicher und nutzloser Ballast bewertet werden, der aus rein formalen Gründen während eines Hochschulstudium mitgeschleppt werden muss, halte ich für einen gefährlichen Nebeneffekt des Beharrens auf Begriffen, deren praktische Anwendbarkeit als fraglich gelten muss.

Um eine gewisse Ausgewogenheit des Textes zu gewährleisten, möchte ich an dieser Stelle auf zwei Fälle hinweisen, die mir in meiner pädagogischen Praxis als sinnvolle Anwendungsdomänen des Morphembegriffes aufgefallen sind:

(a) Das Suffix - ung indiziert eindeutig Femininum und kann daher zur Formulierung von Regeln zur Genuszuweisung verwendet werden. Voraussetzung der erfolgreichen Anwendung einer solchen Regel ist allerdings, dass der Lerner das Suffix - ung von anderen, zufällig ung ergebenden Phonemfolgen unterscheiden kann, vgl. Nutzung (f) $\times$ Ursprung $(\mathrm{m})$. Hier könnte der Hinweise, die Form Nutzung zerfalle in zwei morphematische Komponenten (Nutz-ung), von denen die eine das gebundene Morphem /ung/ darstelle, wohingegen Ursprung kein Morphem /ung/, sondern vielmehr ein Allomorph des Basismorphems/spring/ enthalte, nachvollziehbar Aufklärung schaffen.

(b) Eine ähnliche Regel zur Genuszuweisung lässt sich mit Bezug auf das Suffix er formulieren, mit dessen Hilfe maskuline nomina agentis abgeleitet werden (Arbeiter $<$ arbeit-, Leser < les-, Retter < rett-). Vermeintliche "Ausnahmen“ von dieser Regel wie etwa Butter (f) oder Fenster (n) ließen sich dann dadurch erklären, dass in diesen Wortformen gerade keine Realisierung des Morphems -er ,Agens' vorliegt.

(a) und (b) stützen sich auf Einsichten in die Strukturierung von sprachlichen Formen, deren Vermittlung unbestritten ein Lernziel des sprachwissenschaftlichen Unterrichts im Rahmen eines Hochschulstudiums sein sollte. Mir scheint allerdings, dass solche Einsichten auch unter Verwendung von theoretisch neutralen Termini wie "Suffix“ oder "Stamm“ vermittelbar sind, die nicht notwendigerweise auf die strukturalistische Morphemanalyse rekurrieren.

\section{Notwendigkeit für die Benutzung von DaF-Hilfsmitteln}

\subsection{Einleitende Überlegungen}

Im vierten Abschnitt des vorliegenden Artikels möchte ich prüfen, ob Studierende für das Lehramt Deutsch an Grundschulen in Tschechien den Morphembegriff während des Studiums oder später in der Berufspraxis - für ihre selbständige Arbeit mit Fachliteratur benötigen.

Natürlich lässt es sich nicht leugnen, dass der Begriff „Morphem“ in der linguistischen Fachliteratur verwendet wird. Diese Aussage muss allerdings durch zwei Zusatzbemerkungen weiter qualifiziert werden: 
(a) Wie wir am Ende des 2. Abschnittes gesehen haben, wird der Begriff "Morphem" in der heutigen Fachliteratur in einer Fülle von unterschiedlichen, manchmal geradezu entgegengesetzten Bedeutungen verwendet. Es ist daher illusorisch, Studierende durch die Einführung des strukturalistischen Morphembegriffes in Seminarübungen generell auf die Lektüre linguistischer Texte vorbereitet zu können. Nimmt der angehende Germanist Fachliteratur zur Hand, in der der Begriff „Morphem“ “verwendet wird, muss er die Bedeutung dieses Terminus in jedem Fall aus dem Zusammenhang der Darstellung im Text erschließen bzw. aus seinem Vorwissen über den der jeweiligen Arbeit zugrunde liegenden theoretischen Rahmen ableiten. Im Unterricht in den Seminaren zu suggerieren, es gäbe die eine, „richtige“ Morphemdefinition, erweist sich angesichts dieser Forschungslage als kontraproduktiv und fordert spätere Missverständnisse geradezu heraus.

(b) Hinsichtlich des Profils der in Tschechien angebotenen Studiengänge für Lehramt an Grundschulen ist es äußerst unwahrscheinlich, dass die Absolventen solcher Studiengänge mit linguistischer Primärliteratur in Kontakt kommen. Sowohl beim Abfassen von Hausarbeiten als auch beim Nachschlagen in der späteren Berufspraxis an den tschechischen Grundschulen ist der Normalfall viel eher der, dass auf Handbücher mit DaF-Ausrichtung oder popularisierende, zusammenfassende Darstellungen zurückgegriffen wird. In einer Betrachtung zur Nützlichkeit des Morphembegriffes in der Deutschlehrerausbildung muss also das Hauptaugenmerk auf solchen Publikationen liegen, die von Studierenden und Absolventen dieser Ausbildungsrichtung erwartungsgemäß konsultiert werden. Ich kann zwar an dieser Stelle keine umfassende Auswertung der entsprechenden Literatur vorlegen, möchte aber zumindest stichprobenartig vier repräsentative Werke auf den Gebrauch des Morphembegriffes hin untersuchen. Bei diesen vier Werken handelt es sich um: Beneš et al. (2005), DUDEN (2009), Helbig/Buscha (1998), und Thieroff/Vogel (2009).

\subsection{Der Morphembegriff bei Beneš et al. (2005)}

Bei Beneš et al. (2005) handelt es sich um eine in Tschechien bzw. der ehem. Tschechoslowakei erschienene Grammatik des Deutschen mit langer Tradition, die seit 2005 vom Fraus-Verlag in Pilsen in einer neu bearbeiteten Version herausgegeben wird. Das morphologisch relevante Kapitel erscheint unter der Überschrift "Tvarosloví" (Formenlehre, S. 41). Schon aus der Benennung ist ersichtlich, dass die Grammatik vom Wort als Grundeinheit ausgeht und (in lateinischer Tradition) die Veränderungen der Wortform („ohýbání slov“, d. h. ,Beugung") registriert, ohne eine Analyse der internen Wortstruktur vorzunehmen. Der Begriff „Morphem“ ist in einem so verstandenen System natürlich überflüssig (vgl. dessen Fehlen im Register, S. 283f.). Aus Gründen der Deutlichkeit werden die Endungen in den Paradigmen dennoch graphisch hervorgehoben (z. B. S. 59: des Mann-es) und mit 
dem trad. Begriff „koncovka“ (Endung) bezeichnet. Die Frage, wie eine „Endung“ im Sinne des Strukturalismus zu analysieren wäre (ein Morphem, mehrere kumulative Morpheme, Allomorphe desselben Morphems oder verschiedener Morpheme bzw. phonologische Varianten ein und desselben Morphs), ${ }^{1}$ wird nicht aufgeworfen. Der Benutzer dieser Grammatik kommt also völlig ohne Vorkenntnisse aus dem Bereich der Morphematik aus.

\subsection{Der Morphembegriff in der Duden-Grammatik (2009)}

Die Grammatik, die im Rahmen der Duden-Reihe erscheint (DUDEN 2009), kann wohl - wie alle Bände der Reihe - als Standard-Nachschlagewerk für die breitere Öffentlichkeit bezeichnet werden. Der Morphembegriff wird gleich am Anfang der Darstellung in $\$ 25$ (S. 38) unter Bezugnahme auf die übliche Definition (,[... die kleinsten bedeutungstragenden Einheiten") eingeführt, dient an dieser Stelle aber in erster Linie der Abgrenzung zur Silbe. Im weiteren Verlauf der Darstellung, insbesondere in den morphologisch relevanten Teilbereichen der Grammatik (d. h. Flexion, $\$ 204 f f$., und Derivation, $\$$ 978ff.), wird der Begriff „Morphem“ dann praktisch nicht mehr verwendet. Hinsichtlich der Flexion (S. 135f.) werden verschiedene „Flexionsmittel“ unterschieden, zu denen neben der Suppletion und der Modifikation (,innere Abwandlung“) die „Flexionssuffixe“ gerechnet werden. Die Begriffe „Suffix“, „Präfix“, „Affix“ bzw. auch „Endung“ (z. B. \$ 278, S. 180, im Kontext der Pluralbildung bei den Substantiven) kommen dann auch weiterhin durchgehend vor. Als komplementärer Begriff dient "Stamm“: „Wenn Wörter flektiert werden, bildet deren Stamm die Grundlage“ (DUDEN 2009: 135, Herv. im Original). Auch bei der Darstellung der Wortbildung werden durchgängig die traditionellen Termini „Stamm“ und „Affix“"verwendet, wobei einleitend nur kurz auf die eingangs gegebene Definition von „Morphem“ in $\$ 25$ verwiesen wird:

Unter Wortbildungsmitteln (auch: Wortbildungseinheiten, Wortbildungselementen) werden solche sprachlichen Einheiten zusammengefasst, die als Ausgangseinheiten, als ,Bausteine' für neue Wörter fungieren können. Typischerweise sind das (a) Wörter bzw. Wortstämme (freie Morpheme, vgl. $\$ 25$ ) und (b) Wortbildungsaffixe (gebundene Morpheme). (DUDEN, $\$ 979$, S. 650).

Fragen der Allomorphie oder der Bestimmung des Inhalts von lautlichen Ketten, die für deren Morphemstatus ausschlaggebend wären, werden nirgends diskutiert.

1 Vgl. Čermák (2001: 122), der sich - allerdings ohne auch nur einen einzigen Grund anzuführen - gegen die Identifizierung von „Morphem“ und „Endung“ verwahrt: „Morfém - flektivní ( $\neq$ koncovka)“. Dass die graphische Hervorhebungen der Endungen in Beneš et al (2005) keine linguistische Analyse anzeigen, sondern nur die Einprägung der Formen erleichtern soll, ist z. B. bereits daraus ersichtlich, dass dem Benutzer (S. 114) unterschiedslos zwei verschiedene Analysen für die Präteritumsformen angeboten werden (er antwort-ete $\times$ er antwortet-e), von denen sie/er je nach eigener Vorliebe die einleuchtendere auswählen kann. 
Dagegen enthält die Darstellung einen expliziten Hinweis darauf, dass vom Konzept des Morphems (trotz dem verbalen Bekenntnis zu diesem Konzept in $\$ 25$ ) abgerückt wird: „Hinsichtlich des semantischen Gehalts der Wortbildungsaffixe hat sich in jüngerer Zeit die Auffassung durchgesetzt, dass sie in Verbindung mit ihrer Basis eher eine oder mehrere Bedeutungen signalisieren, als dass sie selbst darüber verfügen" (DUDEN 2009: 656). Wenn Derivationsaffixe aber selbst über keine Bedeutung verfügen, sind sie laut strukturalistischer Morphemdefinition keine Morpheme.

Insgesamt lässt sich sagen, dass die Duden-Grammatik (trotz einer kurzen Referenz in $\$ 25$ ) ohne den Morphembegriff auskommt. Weder wird er bei der Darstellung der Fakten der deutschen Morphologie verwendet, noch sind Fragen der morphematischen Analyse, wie ich sie in Abschnitt 3 unter (a)-(j) angeschnitten habe, Gegenstand der Darstellung.

\subsection{Der Morphembegriff bei Helbig/Buscha (1998)}

Eine der einflussreichsten Grammatiken für den Fremdsprachenunterricht ist wohl Helbig/Buscha (1998). Helbig/Buscha (1998) verwenden den Morphembegriff zwar hie und da (z. B. S. 27), für das Verständnis der Ausführungen zur Deklination und Konjugation ist er allerdings nicht erforderlich. Die Darstellung beruht im Prinzip auf einem traditionell aufgefassten Wort-und-Paradigma-Modell, das die Wortformen und deren Funktion in den Vordergrund stellt, nicht die interne morphematische Strukturierung dieser Formen. Zu Zwecken der Hervorhebung der grammatischen Marker wird praktisch überall der neutrale Begriff „Endung“, ggf. auch „Präfix“ (z. B. ge- beim Partizip, S. 111) oder „Suffix“(z. B. -er beim Komparativ der Adjektive, S. 304) verwendet. Häufig bleiben die Marker im Text aber völlig ohne Benennung und werden nur (in Kursivschrift) in ihrer äußeren Form angeführt (z. B. S. 110: „durch Anhängen von - $d^{\prime \prime}$, zur Bildung des Partizip I). Einige Beispiele sollen das Gesagte weiter illustrieren:

Zur Konjugation (S. 26) heißt es etwa: „Der Indikativ des Präsens wird bei regelmäßigen und unregelmäßigen gebildet, indem an den Stamm des Verbs folgende Personalendungen angefügt werden." Ohne Bezeichnung bleibt zunächst das Bildungselement -te- (,[...] indem zwischen Stamm und Personalendung ein -te- eingefügt wird“, S. 27), im klein gedruckten Begleitkommentar wird dann von einem "präteritalem Morphem“ gesprochen. Im weiteren Verlauf des Textes (z. B. auf S. 35) ist-te-dagegen einfach ein „Suffix“.

„Endung" ist vermutlich der am häufigsten verwendete Begriff, wie z. B. auf S. 36 („Personalendung“), S. 105 („Infinitivendung“), S. 237 (Endungen zur Bezeichnung der Kasusendungen beim Substantiv), S. 241 (Substantive „ohne Endung“ im Plural) oder S. 300 (,volle Endungen“ beim Adjektiv). Sporadisch werden aber auch noch andere Termini verwendet (z. B. S. 239: „das Flexionskennzeichen - $n$ “ beim Dat. Pl. der Substantive). 
Wie bereits bei den beiden vorher betrachteten Werken fehlt auch bei Helbig/ Buscha (1998) jede Auseinandersetzung mit Fragen der morphematischen Analyse. So werden etwa auf S. 28f. die Kategorien des finiten Verbs besprochen, ohne zu diskutieren, ob es sich bei den entsprechenden Markern um „Morpheme“ bzw. um ein einziges (kumulatives) oder mehrere verschiedene (teilw. ausdruckseitig leere) Morpheme handelt. ${ }^{1}$ Der Ablaut bei starken Verben wird (S. 33) als Änderung des Stammvokals eingeführt; ob eine Vokaländerung als „Morphem“ zu betrachten ist, wird jedoch nicht erörtert.

Dass der Begriff, Morphem “in Helbig/Buscha (1998) praktisch nicht vorkommt, ${ }^{2}$ erscheint mir nur verständlich: Die Darstellung ist nämlich in erster Linie an den praktischen Bedürfnissen des Fremdsprachenunterrichts ausgerichtet und hat zum Ziel, nicht muttersprachlichen Benutzern die richtige Formenbildung vor Augen zu führen sowie die richtige Verwendung der Formen als Ganzes, nicht jedoch die Abbildung der Merkmale oder Funktionen auf die einzelnen Strukturelemente einsichtig zu machen. Für ihre Zwecke halten die Autoren die Verwendung des Begriffes „Morphem“ offenbar für überflüssig, weswegen der Begriff auch nicht im Sachregister (S.721ff.) erscheint.

\subsection{Der Morphembegriff bei Thieroff/Vogel (2009)}

Das bunteste Bild bietet aus terminologischer Sicht die Broschüre von Rolf Thieroff und Petra Vogel (Thieroff/Vogel 2009), die eine komprimierte Darstellung der flexionsmorphologischen Grundfakten des Deutschen für Germanistikstudenten bietet. Offenbar hängt mit dieser Zielgruppe (Hochschulstudenten im deutschsprachigen Raum, nicht in erster Linie Lerner des Deutschen als Fremdsprache) auch der erhöhte terminologische Aufwand zusammen, den die Autoren bei ihrer Darstellung treiben. Die traditionellen Termini „Stamm“, „Suffix“ und „Endung" finden sich in bunter Mischung neben dem Begriff „Morphem“ und diversen Zusammensetzungen („Stammmorphem“, S. 8; „einmorphemige Stämme“, S. 11; „Person-Numerus-Morphem“, S. 14, neben „Person-NumerusEndung", S. 13).

Die parallele Verwendung von Begriffen unterschiedlicher Provenienz lässt sich schön an folgendem Zitat (S. 11) demonstrieren: „Liegt ein komplexes Verb mit Präfix vor, also mit einem unselbständigem [sic] Morphem, erfolgt die Partizipbildung nur über das Suffix - $t$ bzw. -en [...]“. Welche Funktion hier die doppelte Benennung desselben Elements durch zwei unterschiedliche Termini haben soll, ist mir nicht klar geworden.

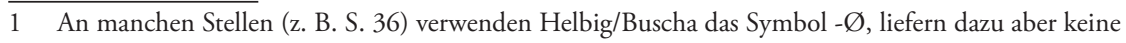
morphematische Begründung, sondern sprechen lediglich von „Endungslosigkeit“.

2 Eigentlich ist die bereits zweimal zitierte Anmerkung auf S. 27 die einzige Textstelle mit Morphembezug, die ich bei überfliegender Durchsicht des Textes finden konnte. Im Sachregister wird der Begriff „Morphem“ gar nicht aufgeführt. 
Außer den traditionellen Termini findet in Thieroff/Vogel (2009) auch der Begriff „Marker“ bzw. „Markierung“ Anwendung, der sich in neueren Arbeiten immer stärker durchzusetzen beginnt. An manchen Stellen kommt dabei der Verdacht auf, dass „Morphem“ lediglich als traditionelleres Synonym zu dem „moderneren“ Terminus „Marker“ verwendet wird. Wenn es z. B. auf S. 14 heißt, dass „[...] in diesem Morphem [d. h. dem Präteritumssuffix -te-] gleichzeitig die Merkmale Präteritum und Konjunktiv verwirklicht" seien, kann hier eigentlich nicht von einem strukturalistischen Morphem die Rede sein, das ja - im Unterschied zum Marker - nicht die materielle Realisierung von morphosyntaktischen Merkmalen, sondern als doppelseitiges Zeichen die un mittelbare Einheit solcher Merkmale mit ihrer (abstrakten) Ausdrucksform darstellt. Als abstrakte Einheit des Sprachsystems realisiert ein Morphem überhaupt nichts, wird aber seinerseits (auf einer niedrigeren Sprachebene) durch eine bestimmte Phonemfolge repräsentiert. Offenbar wird in der zitierten Passage der strukturalistische Begriff „Morphem“ mit poststrukturalistischen Konzeptionen von morphosyntaktischen Merkmalen und deren Realisierungen kurzgeschlossen. Zur terminologischen (und konzeptuellen) Klärung trägt ein solches Verfahren wohl eher weniger bei.

Es lässt sich vermuten, dass die Darstellung in Thieroff/Vogel (2009) auf Studierende, die noch nie mit dem Morphembegriff in Berührung gekommen sind, verwirrend und letztendlich auch abschreckend wirkt. Durch die permanente Verwendung von unterschiedlichen Termini zur Bezeichnung derselben Sachverhalte sollte aber andererseits jeder, der sich von der geballten Ladung fremdsprachiger Terminologie nicht gleich abschrecken lässt, in der Lage sein, das Gemeinte aus dem Kontext zu erschließen.

Nach Durchsicht von einigen für den DaF-Unterricht in Tschechien relevanten Publikationen komme ich somit zu dem Schluss, dass in drei der vier betrachteten Arbeiten (Beneš et al. 2005; DUDEN 2009; Helbig/Buscha 1998) der Morphembegriff keine oder nur eine äußerst marginale Rolle spielt. Die in diesen Arbeiten enthaltenen Darstellungen der deutschen Morphologie sollten demnach auch für Leser verständlich sein, die keine Vorkenntnisse in der morphematischen Analyse haben. Eine Publikation (Thieroff/Vogel 2009) macht dagegen ausgiebig (und zudem nicht besonders kohärent) Gebrauch von dem Begriff „Morphem“. Ein in der morphematischen Analyse ungeschulter Leser muss sich hier für das Textverständnis auf die parallel benutzten, traditionellen Termini stützen.

\section{Der Begriff "Morphem“ und begriffliche Alternativen für den DaF- Unterricht}

Ich will nun versuchen, das Fazit der in diesem Artikel angestellten Überlegungen zu ziehen. In Abschnitt 1 habe ich versucht, die ursprüngliche Motivation für die Einführung des Begriffes „Morphem“ zu rekonstruieren. Der Begriff „Morphem“ entspringt ursprünglich einem semiotischen Verständnis von Sprache, demgemäß 
erst die unauflösliche Verbindung von Ausdruck und Inhalt sprachliche Objekte konstituiert. In Abschnitt 2 habe ich gezeigt, dass dieses Verständnis (auf sublexikalische Einheiten angewandt) nicht unproblematisch ist, weswegen sich zahlreiche linguistische Schulen von dem Morphembegriff abgewendet haben bzw. den Terminus „Morphem“ in anderen, nicht der klassischen Definition entsprechenden Bedeutungen verwenden. In Abschnitt 3 habe ich einige elementare Daten des Deutschen unter morphematischen Gesichtspunkten analysiert und dabei festgestellt, dass diese Analyse schwerwiegende Probleme aufwirft und Fremdsprachenlerner in den meisten Fällen keinen Gewinn aus solchen Analysen ziehen können. Wie die Übersicht in Abschnitt 4 zeigt, ist der Begriff „Morphem“ auch nicht Voraussetzung des Verständnisses der Darstellungen in vielen DaFrelevanten Handbüchern und Hilfsmitteln.

Daraus ergibt sich für mich eine klare Schlussfolgerung: Für den Unterricht in Studiengängen, die der Ausbildung von Grundschullehrern an allgemeinbildenden Schulen (z. B. in Tschechien) dienen, ist die Einführung des Begriffes „Morphem“ überflüssig. Für die Zwecke der Ausbildung solcher Lehrer und Lehrerinnen kommt man mit den traditionellen Begriffen „Stamm“, „Suffix“ oder „Präfix“ aus, wobei auch die für die deutsche Morphologie sehr wichtige modifizierende Markierung problemlos einbezogen werden kann. Notwendige Einsichten in die Strukturierung von Wörtern (wie etwa die Erkenntnis, dass das Segment -er in Leser und Butter oder Fenster eine andere Rolle spielt) lassen sich auch mit diesem theorieneutralen Begriffsinventar vermitteln. Sofern ein Oberbegriff für alle sprachlichen Mittel zur Markierung von grammatischer Information erforderlich ist, bietet sich der Begriff „Marker" an, wie er etwa bei Wurzel (2000) oder (in Anlehnung daran) bei Janakiev (2010) auch für den Fremdsprachenunterricht vorgeschlagen wird. Im Übrigen wird der Begriff „Marker“ in diesem Sinne bereits von Autoren aus dem Umfeld des Prager Zirkels verwendet (Trnka 1932) und stellt daher keine terminologische Innovation dar.

Zwar sieht der Autor dieser Zeilen hinsichtlich morphematischer Fragen keinen Handlungsbedarf für den Gesetzgeber, im Unterschied etwa zur „Gesellschaft gegen den Missbrauch von Morphemen“, die (freilich nicht ganz ernst gemeint) ein Verbot des Begriffes „Morphem“ anregt (zit. in Mugdan 1986: 40); eine freiwillige Selbstbeschränkung sollten sich Autoren von Skripten für Lehramtsstudenten meiner Meinung nach aber schon auferlegen, schon damit Sprachwissenschaft in den entsprechenden Einführungskursen nicht zur sinnentleerten Pflichtübung gerät.

\section{Literaturverzeichnis}

Anderson, Stephen R. (1982): Where's Morphology? In: Linguistic Inquiry 13/4/1982. S. 571-612.

Anderson, Stephen R. (1992): A-Morphous Morphology. Cambridge, Cambridge University Press. 
Aronoff, Mark (1976): Word formation in generative grammar. Cambridge/Massachusetts und London, MIT Press.

Aronoff, Mark / Fudeman, Kirsten (2005): What is Morphology? Malden, Oxford und Carlton, Blachwell Publ.

Baudouin de Courtenay, J. (1880): Подробная программа лекций в 1877-1878 учебном году (продолжение). In: Известия Императорскаго Казанскаго Университета 16/2/1880. S. 269-480.

Beneš, Eduard / Jungwirth, Karel / Kouřimská, Milada / Zapletal, Štěpán (2005): Praktická mluvnice némčiny. Prepracované a aktualizované vydání oblibené mluvnice. Plzeň, Nakladatelství Fraus.

Bierwisch, Manfred (1975): Syntaktische Merkmale in der Morphologie: generelle Probleme der sogenannten pronominalen Flexion im Deutschen. In: Kiefer, Ferenc (Hg.): Morphologie und generative Grammatik. Frankfurt a. M., Athenaion. S. 1-55.

Bierwisch, Manfred (1989): Event nominalizations: proposals and problems. In: Motsch, Wolfgang (Hg.): Wortstruktur und Satzstruktur. (= Linguistische Studien, Reihe A, Arbeitsbericht 194). Berlin, Akademie der Wissenschaften der DDR. S. 1-71.

Bittner, Andreas (1996): Starke, schwache'Verben - schwache, starke'Verben. Deutsche Verbflexion und Natürlichkeit. Tübingen, Stauffenburg.

Bloomfield, Leonard (1933): Language. New York, Holt.

Bloch, Bernard (1947): English verb inflection. In: Language 23/4/1947. S. 399-418.

Bondarko, Aleksandr Vladimirovič (1984): Функциональная грамматика. Ленинград, Наука.

Bußmann, Hadumod (1990): Lexikon der Sprachwissenschaft. 2. Aufl. Stuttgart, Alfred Körner.

Chomsky, Noam (1957): Syntactic structures. S'-Gravenhage, Mouton. Zitiert nach der französischen Übersetzung von Michel Braudeau. Paris, Editions du Seuil, Collection Points, Bd. 98.

Chomsky, Noam (1965): Aspects of the theory of syntax. Cambridge/Massachusetts, MIT Press.

Clément, Danièle (1996): Linguistisches Grundwissen. Opladen, Westdeutscher Verlag.

Čermák, František (2001): Jazyk a jazykověda. Přhled a slovník. Praha, Karolinum.

Čermák, František (2011): Morfématika a slovotvorba češtiny. 2. Aufl. Praha, Lidové noviny.

DUDEN (2009): Wermke, Matthias / Kunkel-Razum, Kathrin [u. a.] (Hg.): Duden Band 4:

Die Grammatik. 8. Aufl. Mannheim [u.a.], Dudenverlag.

Eisenberg, Peter (2006): Grundriss der deutschen Grammatik, Band 1: Das Wort. 3. Aufl. Stuttgart und Weimar, Metzler.

Erben, Johannes (1972): Deutsche Grammatik. Ein Abriss. 11. Aufl. München, Hueber.

Ernst, Peter (2004): Germanistische Sprachwissenschaft. Basel, Wien [u. a.], UTB.

Fleischer, Wolfgang / Barz, Irmhild (2007): Wortbildung der deutschen Gegenwartssprache. 3. Aufl. Tübingen, Max Niemeyer.

Geislerová, Olga (2007): Einführung in die Morphologie der deutschen Sprache. Studienmaterial für Lehramtsstudenten. Brno, Pedagogická fakulta Masarykovy univerzity.

Helbig, Gerhard / Buscha, Joachim (1998): Deutsche Grammatik. Ein Handbuch für den Ausländerunterricht. 18. Aufl. Leipzig, Berlin, München [u. a.], Langenscheidt/Verlag Enzyklopädie.

Heringer, Hans Jürgen (2009): Morphologie. Paderborn, UTB/Wilhelm Fink.

Hudson, Richard (2007): Language networks. The new word grammar. Oxford, Oxford University Press. 
Hockett, Charles F. (1947): Problems of morphemic analysis. In: Language 23/4/1947. S. 321-43.

Hockett, Charles F. (1961): Linguistic elements and their relations. In: Language 37/1/1961. S. 29-53.

Janakiev, Angrit (2010): Ausgewählte Probleme der Flexion der Wortartkategorie Verb in der DaF-Lehrerausbildung. In: Brünner Hefte zu Deutsch als Fremdsprache 3/2/2010. S. 181-195.

Janíková, Věra (2012) Fortbildung als Chance zur Qualitätssicherung des fremdsprachlichen Unterrichts. In: Konzeptionen und Strategien lebenslangen Lernens im internationalen Kontext. Sankt-Petersburg, SPB APPO. S. 96-100.

Káňa, Tomáš (2006): Úvod do studia némeckého jazyka. Struktura prédmètu na PdF MU. Brno, Pedagogická fakulta Masarykovy univerzity.

Komárek, Miroslav (2006): Př́spévky k české morfologii. 2. Aufl. Olomouc, Periplum.

Lamb, Sydney M. (1966): Outline of Stratificational Grammar. Washington, Georgetown University Press.

Leskien, August (1910): Handbuch der Altbulgarischen Sprache. Grammatik - Texte - Glossar. 5. Aufl. Heidelberg, Carl Winter Universitätsverlag.

Luschützky, Hans Christian (2000): Morphem, Morph und Allomorph. In: Booij, Geert / Lehmann, Christian / Mugdan, Joachim (Hg.): Morphologie. Ein internationales Handbuch zur Flexion und Wortbildung. Halbband 1. (= Handbücher zur Sprach- und Kommunikationswissenschaft, Bd. 17). Berlin und New York, de Gruyter. S. 451-462.

Matthews, Peter H. (1972): Inflectional Morphology. A Theoretical Study Based on Aspects of Latin Verb Conjugation. Cambridge/Großbritannien, Cambridge University Press.

Mugdan, Joachim (1986): Was ist eigentlich ein Morphem? In: Zeitschrift für Phonetik, Sprachwissenschaft und Kommunikationsforschung 39/1/1986. S. 29-43.

Nida, Eugene A. (1948): The Identification of Morphemes. In: Language 24/4/1948. S. 414-448.

Nübling, Damaris (2008): Was tun mit Flexionsklassen? Deklinationsklassen und ihr Wandel im Deutschen und seinen Dialekten. In: Zeitschrift für Dialektologie und Linguistik 75/3/2008. S. 282-330.

Nübling, Damaris / Dammel, Antje / Duke, Janet / Szczepaniak, Renata (2010): Historische Sprachwissenschaft des Deutschen. Eine Einführung in die Prinzipien des Sprachwandels. 3. Aufl. Tübingen, Narr Francke Attempto Verlag.

Plungjan, Vladimir Aleksandrovič (2003): Общая морфология. Введение в проблематику. 2. Aufl. Москва, УРСС.

Römer, Christine (2006): Morphologie der deutschen Sprache. Tübingen und Basel, A. Francke.

Ruwet, Nicolas (1967): Introduction à la grammaire générative. Paris, Librairie Plon.

Saussure, Ferdinand de (1931): Cours de linguistique générale. Publié par Charles Bally et Albert Sechehaye. 2. Aufl. Paris, Payot. (1. Aufl. 1916.)

Stump, Gregory T. (1991): A Paradigm-Based Theory of Morphosemantic Mismatches. In: Language 67/4/1991. S. 675-725.

Stump, Gregory T. (2007): Inflection. In: Spencer, Andrew / Zwicky, Arnold, M. (Hg.): The handbook of morphology. 4. Aufl. Malden [u.a.], Blackwell. S. 13-43.

Šimečková, Alena (2004): Úvod do studia jazykovědné germanistiky. Praha, Karolinum.

Thieroff, Rolf / Vogel, Petra M. (2009): Flexion. (=Kurze Einführungen in die germanistische Linguistik, Band 7). Heidelberg, Universitätsverlag Winter.

Trnka, Bohumil (1932): Some thoughts on structural morphology. In: Charesteria Guil. Mathesio. Praha. Zit. nach der russ. Übersetzung (Neskolko myslej o strukturnoj 
morfologii). In: Kondrašova, N. A. (Hg.): Pražskij lingvističeskij kružok. Sbornik statej. Moskva, Progress, 1967. S. 266-271.

Vendryès, J. (1921): Le langage. Introduction linguistique à l'historie. Paris, La Renaissance du Livre.

Welke, Klaus (2002): Deutsche Syntax funktional. Perspektiviertheit syntaktischer Strukturen. Tübingen, Stauffenburg.

Wurzel, Wolfgang Ullrich (2000): Der Gegenstand der Morphologie. In: Morphologie. Ein internationales Handbuch zur Flexion und Wortbildung. Halbband 1. (= Handbücher zur Sprach- und Kommunikationswissenschaft, Bd. 17). Berlin und New York, de Gruyter. S. $1-15$.

Ziková, Markéta (2004): Morfologie a vnitřní struktura slova. In: Karlík, Petr / Pleskalová, Jana (Hg.): Život s morfémy. Sborník studii na počest Zdenky Rusinové. Brno, Masarykova univerzita. S. 185-192. 